\title{
L. J. Müller, Sound und Sexismus. Geschlecht im Klang populärer Musik. Eine feministisch- musiktheoretische Annäherung, Hamburg: Marta Press 2018
}

Schlagworte/Keywords: gender studies; Genderforschung; Klanganalyse; performance analysis; Performanceanalyse; popular music research; Popularmusikforschung; sexism; Sexismus; sound analysis

In jüngerer Zeit finden im musikwissenschaftlichen Diskurs zunehmend Stimmen Gehör, die sich für performance analysis als Komplement zur score analysis stark machen, also für eine Methode der Musikanalyse, die nicht das Notat, sondern das real Erklingende als sdie $\mathrm{Mu}$ sikı versteht und untersucht, was Musizierende tun, "to give meaning to sounds «. ${ }^{1}$ Man muss Notentextanalyse nicht als "Plato's Curse « ${ }^{2}$ empfinden, um von der Idee fasziniert zu sein, dass anstelle eines schriftlich fixierten Notenbilds tatsächliches Klanggeschehen zum Gegenstand analytischer Betrachtung wird, zumal bestimmte musikalische Stile ohne den Einbezug der Dimension 'Klang، überhaupt nicht adäquat erfasst werden können. Dies gilt etwa für den Belcantostil der italienischen Oper des 19. Jahrhunderts, dessen Realisierung auf die Berücksichtigung einer Unzahl performativer Stilmittel angewiesen ist, die sich teils nur schwer - und vor allem nicht in Details ihrer konkreten Ausführung - notieren lassen. ${ }^{3} \mathrm{Ge}$ nerell sind von der geringen Passgenauigkeit an Notentexten entwickelter Analysekategorien Musikstile betroffen, deren Identität substanziell von klangfarblichen Wirkungen der menschlichen Stimme abhängt. So wurden besonders engagierte Versuche, Kategorisierungen vokaler Klangfarben vorzunehmen, im Schrifttum zur Popularmusik unternommen - sicherlich nicht zufällig, aus mindestens drei Gründen: Erstens

1 Leech-Wilkinson 2009, Kap. 2, Abs. 29: „This is something that the analysis of performance can do rather well, taking the performance as the music, and examining the kinds of things that performers do to give meaning to sounds. "

2 Vgl. Cook 2013, 8-32 (»Plato's Curse«).

3 Vgl. Berne 2008; Sprau i. V. handelt es sich um einen Forschungsbereich, der es regelmäßig mit dem song als Formeinheit, also mit einer explizit vokalen Ausprägung von Musik zu tun hat; zweitens liegen als Analyseobjekte häufig nicht Notentexte, sondern Aufnahmen und damit ein konkret gegebenes Klanggeschehen vor; drittens und damit zusammenhängend wird der ssound in vielen Spielarten der Popularmusik als zentrale strukturelle Kategorie verstanden. ${ }^{4}$ Freilich stellen sich der Erarbeitung objektiver Kategorien zur höranalytischen Erfassung stimmlicher Klangfarben Hindernisse in den Weg. Differenzierte Kriterienkataloge wurden in der Gesangspädagogik vorgelegt, sind aber nachvollziehbarerweise stark an der sängerischen Klangproduktion orientiert und nicht primär auf körperexternhöranalytische Rezeptionsmodi ausgerichtet. ${ }^{5}$ Auch hat die objektivierende Klassifizierung menschlicher Stimmfarben in besonderer Weise mit subjektiven Assoziationen und Konnotationen zu tun. So stellt Jennifer Walshe ihren "Genealogien populärer Klangfarben« (d. h. Stimmfarben) eine Einleitung voran, welche die "Subjektivität« betont, mit der die menschliche Hörwahrnehmung stimmlichen Klangfarben begegne: »die Beziehungen zwischen den disparaten vokalen Klangfarben der populären Musik [...] können nicht systematisch klassifiziert werden«, jedenfalls nicht auf Basis »der Objektivität eines distanzierten Beobachters «. ${ }^{6}$

Es ist daher für die Fachcommunity von Bedeutung, dass L. J. Müller 2018 im Hamburger Verlag Marta Press ein Buch veröffentlicht hat, das sich laut Untertitel als »feministisch-musik-

4 Vgl. Smith 2008, 120; Walshe 2010, 57.

5 Vgl. etwa Sadolin 2013; Soto-Morettini 2014.

6 Walshe 2010, 58. 
theoretische Annäherung" an den "Klang populärer Musik«, d. h. den Klang der menschlichen Stimme in populärer Musik, versteht. Das Buch ist aus einer an der Berliner HumboldtUniversität betreuten musikwissenschaftlichen Abschlussarbeit hervorgegangen (vgl. 11), die den Auftakt zu weiterführenden Studien bildete (vgl. 17). ${ }^{7}$ Der im Untertitel prominent verwendete Musiktheorie-Begriff dürfte vor diesem Hintergrund kaum im Sinne einer akademischfachlichen Zuordnung zu verstehen sein, sondern eher als Signal für einen analytisch differenzierenden Zugang zum Thema IStimmklang, bzw. intersubjektive "Überprüfbarkeit» (64) der Ergebnisse. Dass dabei auch der für die Popularmusikforschung quasi selbstverständliche Blick auf übergeordnete gesellschaftliche Zusammenhänge erfolgt, macht der Gesamttitel der Schrift deutlich: Sound und Sexismus. Geschlecht im Klang populärer Musik. Eine feministisch-musiktheoretische Annäherung. Es geht also in Müllers Studie um die Verbindung kategoriengeleiteter, strukturorientierter Musikanalyse mit einem gesamtgesellschaftlich relevanten Thema. Dass sie 2019 mit dem Buchpreis der International Association for the Study of Popular Music ausgezeichnet wurde, zeigt, dass sie ein interessiertes Publikum gefunden hat. Die folgende Rezension beruht auf einer Lektüre aus fachlich-musiktheoretischer Sicht; neben rein wissenschaftlichen Aspekten steht dabei auch die Frage nach unterrichtspraktischen Anschlussmöglichkeiten in den Disziplinen Analyse und Gehörbildung im Raum.

Der zentrale Teil des Buchs gliedert sich in vier Hauptkapitel, von denen die ersten beiden »Theoretische Grundlagen« (31-55) und methodisches Vorgehen (»Analysewerkzeuge«, 57-102) entfalten, und zwar auf vorbildliche Weise voneinander differenziert wie aufeinander bezogen. Die anderen beiden Kapitel legen konkrete Analysen von Popsongs vor, an denen die Theorie exemplifiziert und die analytische

7 Vgl. Müller 2017 und 2018. Beide Publikationen sind inhaltlich nah mit dem hier besprochenen Buch verwandt und leisten weitere Beiträge zur genderkritischen Songanalyse. Die Songanalyse in Müller 2016 verwendet analoge Strategien; sie zielt im Zeichen kulturwissenschaftlich informierter Rassismuskritik auf Dekonstruktion einer »weißen Perspektive auf populäre Musik« (ebd., 217).
Methodik erprobt werden (»)Echtheit als implizit männliche Norm«, 103-129; »Alternative ästhetische Strategien von Sängerinnen «, 131171). Ausgangspunkt des Forschungsvorhabens ist Müllers Intention, »Sexismus im Klang populärer Musik [...] hörbar und benennbar zu machen", um auf diese Weise fundierte »Kritik" an der »Reproduktion [dieses Sexismus] zu ermöglichen« (181). Der zugrunde gelegte Musikbegriff ist konstruktivistisch: Musik wird nicht als »einfach gegebenes Objekt« (16) betrachtet, sondern als »emotionale und körperliche Erfahrung « (18) des rezipierenden Subjekts. Folgerichtig macht Müller »das hörende Subjekt bzw. den Prozess des Hörens" -»als Teil dessen, was Musik ausmacht « (19) - selbst zum Gegenstand der Untersuchung, entwickelt »Begriffe zur Differenzierung von Unterschieden in der Musikerfahrung", die als "Werkzeug[e] für das eigene Hören « und letztlich für eine »kritische feministische Musikbetrachtung " (181 f.) nutzbar gemacht werden sollen. Von Gepflogenheiten des umfangreich zitierten Forschungsdiskurses setzt sich Müllers Studie ab, wenn sie für einen »Großteil der Forschung zu Geschlecht und Popmusik« eine Auseinandersetzung "vor allem mit Kontexten (Biographien, Musikgeschichte, Diskursen, Subkulturen, Genres, Produktions- und Konsumptionsbedingungen)« (20) konstatiert. Müller selbst richtet den Fokus demgegenüber konsequent auf eine »musikspezifische Dimension« (22), nämlich die »Materialität des Klangs" (24), die zum zentralen Analysegegenstand erwählt und als geschlechtlich kodierte "Struktur (23) interpretiert wird.

\section{THEORETISCHE BASIS}

Die Studie ruht auf vier theoretischen Grundpfeilern. Erstens geht sie gemäß dem von Simone de Beauvoir herleitbaren Konzept des Othering (35 f.) davon aus, dass Männer und Frauen gesellschaftlich ungleich positioniert sind, wobei das Weibliche die Funktion eines sAnderen, gemessen am hegemonial vorgeordneten Männlichen, erfüllt. Zweitens übernimmt Müller das durch Judith Butler etablierte Konzept der "Performativität« des Geschlechtlichen; dieses stellt demnach keine gegebene Größe dar, sondern wird »durch die ständige Anpassung von Körper und Subjekt an 
gesellschaftlich vermittelte Normen [...] laufend in jeder Interaktion als solches re/produzier[t] « (36 f.). Drittens betrachtet Müller Popmusik unter Bezugnahme auf Michel Foucault als "global agierendes Sozialisierungssystem« (46), das »normative Geschlechterbilder gesellschaftlich (mit-)reproduziert« (51). Popsongs wirken demnach als »musikalische Präsentation von Geschlecht« (52), etwa durch »eine hörbare Anpassung [...] des in der Stimme performten Körper-Subjekts an kulturelle Normen« (41). Viertens wird die Rezeption von Popmusik als »mimetische[r] Prozess[] « (53) verstanden; Popsongs prägen demnach durch Korrespondenzen zwischen Musik- und Körpererfahrung die »innere Körperwahrnehmung" ihrer Rezipient*innen bzw. deren "Habitus « (54) im Sinne Pierre Bourdieus und beeinflussen damit letztlich die gesellschaftliche Identitätsbildung des Subjekts. Die Kategorie des Körperlichen ist für den weiteren Verlauf der Untersuchung insofern von zentraler Bedeutung, als sie die Verbindung zwischen gesellschaftlich geformten Kategorien des Geschlechtlichen einerseits und subjektiver Musikerfahrung andererseits herstellt. Diese Verbindung analytisch aufzuschlüsseln, ist Ziel der Untersuchungsmethodik.

\section{METHODIK}

Sechs Analysekategorien werden eingeführt: erstens die "Assoziation « im Sinne eines »inneren Wahrnehmungszustandesı, der durch Musik aufgerufen werden kann « (59). In diesem Zusammenhang reagiert Müllers Studie auf das Desiderat "einer ausgearbeiteten popmusikalischen Semiotik« (61), dem sie durch Ansätze zu einer Semiotik des Stimmklangs begegnet (vgl. 94-102). Die zweite Kategorie ist die »Homologie« zwischen »bestimmten Strukturen der Musik und der sozialen Realität der Hörer_innen « (66; als Beispiel dienen mit Bezug auf Susan McClary Korrespondenzen zwischen musikalischen Spannungsverläufen und "Strukturen « [70] sexuellen Begehrens). Die beiden folgenden Kategorien zielen, da die analytische Anwendung von Assoziation und Homologie stark von subjektiven Kriterien abhängt, auf die methodische Integration des s Subjektiven $a b$, und zwar unter Rückgriff auf Theoreme psychoanalytischer Provenienz: Die
Analyse des »Genotextes" (Julia Kristeva) von Songlyrics soll einen Zugriff auf die "unbewusste sprachliche Prägung des Körpers« (74) ermöglichen; die Erörterung sauditiver Lüstes (in Anlehnung unter anderem an Laura Mulvey) nimmt Rezeptionsphänomene im Zusammenhang mit gehörtem Stimmklang in den Blick, darunter die rezipierende Identifikation mit dem/der Performer*in, das Verschwimmen der "Grenze zwischen Selbst und gehörter Stimme« (84). Für die anschließende Analyse konkreter Songs besonders bedeutsam sind schließlich zwei körperbezogene Kategorien: "sonischer Körper« (88) - eine stabile Kombination von Körpervorstellungen, die aus wiederholter Begegnung mit bestimmten musikalischen Erfahrungen resultiert - und "vokalische[r] Körper - " das Bild, das sich im Hören von der Schallquelle gemacht wird« (93), also die Körpervorstellung, die während des Rezeptionsvorgangs "auf den_die Sänger_in projiziert" (55) wird. Die Kategorie des sonischen Körpers adaptiert ein Konzept von Peter Wicke (vgl. 88), die Idee eines "vocalic body« wurde von Steven Connor geprägt (vgl. 93); beide Körperkonstrukte werden als kulturell geformt begriffen und lassen sich daher auf Korrespondenzen zu gesellschaftlich vermittelten Geschlechterbildern hin untersuchen. Ziel der folgenden Songanalysen ist es daher, »im Popsong die stimmliche Produktion von KörperSubjekten zu analysieren« (93) und auf diese Weise die »Produktion von Geschlecht und Sexismus im Klang populärer Musik« (103) aufzuspüren.

\section{ANALYSEN}

In Übereinstimmung mit dem skizzierten Setting werden Popsongs von Müller nicht als Kompositionen im Sinne abstrakt notierbarer struktureller Zusammenhänge (etwa diastematischer oder rhythmischer Art) untersucht, sondern als "performative Akte« (42), d. h. als über Tonträger verfügbare Performances, die einen konkreten Stimmklang transportieren und damit bestimmte Körpervorstellungen kommunizieren. Die Tonträgeranalysen sind in zwei Kapitel gegliedert. Das erste arbeitet am Beispiel zweier Sänger (Kurt Cobain, Robbie Williams), kongruent zur Theorie des Othering, spezifische Eigenschaften smännlich konnotierten 
Stimmklangs als kulturell etablierte Norm heraus, von der sich Vertreterinnen eines sweiblich konnotierten Klangs durch andersartigen Stimmgebrauch abheben. Entsprechende »[a]lternative ästhetische Strategien « (131) werden dann im darauffolgenden Kapitel anhand von Songeinspielungen der Sängerinnen Kate Bush, Kylie Minogue, Björk und Birdy aufgezeigt. Dafür werden zunächst in der Popularmusikforschung vorfindliche Strategien, die »männliche Rockstimme als Norm« (103) aufzustellen, einer kritischen Durchsicht unterzogen; abermals werden verbreitete Mängel im Methodischen und damit verbundene Schwierigkeiten festgestellt, den »Klang selbst« (106) analytisch in den Griff zu bekommen (vgl. 103-109). Müller selbst erklärt einleuchtend das Merkmal der »Authentizität» (die » sechter Stimme«; 111), das häufig mit der »weißen männlichen Rockstimme« assoziiert erscheint, als "Konstruktion von Männlichkeit", die sich als »performativ erzeugt[er] « (113) Effekt identifikatorischer Rezeption ergibt. Als Stilmerkmale des sängerischen Stimmeinsatzes, die mit diesem "Eindruck von Natürlichkeit» (113) korrelieren, nennt Müller für Kurt Cobain (Smells Like Teen Spirit) die Suggestion von "Anstrengung" in den höheren Lagen, "Geräuschlastigkeit der Stimme» durch einen im Rachen produzierten Frikativ (»zwischen einem $\mathrm{R}$ - und einem $\mathrm{CH}$-Laut«) sowie die Anmutung von "Heiserkeit« (116 f.). Als von Robbie Williams (Feel) eingesetzte Gestaltungsmittel werden »nach unten gleitende[ ] Töne", ein dem »Rufen« ähnlicher Stimmklang, geräuschbetonte Aussprache und der schon bei Kurt Cobain beobachtete "raue[ ] Rachenklang" (123 f.) genannt. Deutlich erkennbar wird in beiden Fällen - bei Kurt Cobain noch mehr als bei Robbie Williams - die Konzeption der sechten Stimmer als Gegenmodell zum »klassischen Gesangsverständnis», also zum »Ideal einer eindeutigen Tonhöhe, mit rein harmonischem Obertonspektrum, weichen Übergängen, ausgehaltenen Vokalen und der klaren Verständlichkeit des Textes« (116). Auf der Ausdrucksebene wird ein Stimmklang diagnostiziert, der durch »das regelmäßige akustische In-Erscheinung-Treten von Körperregionen, [...] die unter der Haut liegen« (»z.B. Rachen, Lunge/Zwerchfell, Kehle«; 124) »das Innere des Körpers betont«, der schmerzhafte Empfindungen bis hin zur
Selbstverletzung signalisiere, außerdem »Dringlichkeit" der Mitteilung (117f.) und "Anstrengung gegen den eigenen Körper « (123). Diese durch den Stimmgebrauch bewirkte Inszenierung interpretiert Müller im Sinne »männliche[r] Performanz « als "selbstbewussten Ausdruck der eigenen somatischen Empfindungen «: Indem "die in der stimmlichen Anstrengung enthaltene Kraft oder der heldenhafte Kampf um die Mitteilung« (125 f.) aktiv ausgestellt würden, finde die »erfolgreiche Produktion des Sängers als somatischem Subjekt, das sich selbst ausdrückt« (129), statt.

Die These zu plausibilisieren, dass das skizzierte »ästhetische Modell der sechten` Stimme [...] verbreitet zur Darstellung von Männlichkeit eingesetzt wird", nicht aber von 'Weiblichkeits, ist die Funktion der vier folgenden Analysen von Perfomances weiblicher Sängerpersönlichkeiten, wobei jede der ausgewählten Sängerinnen ein anderes »Muster weiblicher Stimmpräsentationen « (131) repräsentiert.

- An Kate Bush (Feel it) wird ein »kreatives Spielen oder Experimentieren « (134) mit kontrastierenden Registern, mit stimmlichen Klangfarben wie »Kopfstimme», »Bruststimme", "Kinderstimme« (137) exemplifiziert. Das Timbre funktioniere hier in seiner künstlerisch gezielt eingesetzten Wechselhaftigkeit als »Maske«: »Im Kontrast zur sechten s Stimme zeigt sich das Subjekt hierbei nicht mit seiner Emotionalität in der Stimme, sondern sverstecktı sich hinter offenkundig falschen und künstlichen Stimmklischees" (135 f.). Auf der Ausdrucksebene wird dieser »vielfache und widersprüchliche" (141) Stimmeinsatz als »Produktion von Begehren « (136), als Suggestion von "Verführung" (138) und souveräner Appell zu einer »voyeuristische[n] « (141) Rezeptionshaltung gedeutet.

- Auch bei Kylie Minogue (Can't Get You Out of My Head) wird der systematische Gebrauch verschiedener Stimmgebungseffekte festgestellt, darunter eine als »leicht gepresst und knarrend" (143) beschriebene Geräuschhaftigkeit und starke Behauchung; auch werden nasalierte und stark verlangsamte Aussprache konstatiert. Die Nähe solcher körperlich konnotierter Gestaltungsmittel zu Merkmalen der sechten Stimme wird registriert und als »scheinbar 
unmittelbare[r] Zugang zum Körperinnern « (146) interpretiert; dabei ergebe sich in Verbindung mit elektronischen Editing-Effekten (u. a. Vervielfachung der Stimme, Delay) ein "phantastischer " Gesamteindruck: Der »erzeugte vokalische Körper " erscheine in eine »Phantasiewelt« (145) versetzt, wo er sich als "phantastisches Objekt" (151) »dem begehrenden Interesse anbietet« (147). Insgesamt wirke der Stimmklang bei Minogue als "fetischisiertes Objekt» im Rahmen einer "zutiefst verletzenden Darstellung von Weiblichkeit« (147 f.).

- Das Zusammenspiel von stimmlichen und evident elektronisch generierten Klangeffekten prägt auch den Björk-Song All Is Full Of Love, wobei Müller besonders auf eine wechselseitige Annäherung von Stimmklang und synthetischen Sounds hinweist (vgl. 154 f.). In Verbindung mit weiteren Gestaltungsmitteln, etwa extremer Langsamkeit der Deklamation, ergebe sich eine »künstliche Klanglichkeit", durch die sich die menschliche Stimme »im Fiktiven [...] aufzulösen scheint" (156) und wie die Stimme einer entpersonalisierten $» k l a n g l i-$ chen Umwelt« (158) wirke. Dem daraus resultierenden »mangelnden Subjektstatus der Stimme« attestiert Müller mit Verweis auf Jacques Lacan und "verbreitete kulturelle Bilder, wie der Mutter-als-Umgebung, eine starke Verbindung mit Weiblichkeit« (158).

- Vom Beginn des Songs People Help The People an registriert Müller im Stimmgebrauch der Sängerin Birdy Korrelate des Verletzlichen, etwa "Seufzer «-artige Effekte, "eine Art Zittern der Stimme», dem "Weinen « ähnelnde »Lautgebungen « (161). Im Rahmen einer konsequenten Dramaturgie auf verschiedenen Ebenen der musikalischen Gestaltung wird anschließend ein Gang durch verschiedene Stadien stimmlichen Expressionsverhaltens beobachtet, von einer »an das hörbar stoßweise und unkontrollierte Atmen beim Schluchzen« gemahnenden Artikulation und Atemgestaltung (»Schmerz und Trauer«; 162) über Ausdruckselemente von Verzweiflung (»weinend, schluchzend und schreiend «; 163) bis hin zum klanglichen Äquivalent allmählicher Kräftigung mit »tröstenden« (164) Aspekten. Obgleich Müller hier »somatischen
Ausdruck « ähnlich den »männlichen Beispielen der sechten s Stimme« konstatiert, werden doch, wie schon bei Kylie Minogue, substanzielle Unterschiede zu den Performances von Kurt Cobain und Robbie Williams festgestellt: Für den »Eindruck der 'Echtheit«" sorgen demnach bei Birdy nicht »körperlich hörbare Anstrengung und Anspannung", sondern "das Brechen der Stimme«, also "scheinbarer Kontrollverlust» (165). »Ihre Expressivität wirkt damit weniger wie eine selbstbewusste emotionale Mitteilung, sondern vielmehr wie ein verzweifelter Hilferuf" (165 f.): »Die Gefühle offenbaren sich selbst - und zwar gewissermaßen gegen den Willen des singenden Subjekts. Was hier gegenüber den männlichen Beispielen fehlt, ist die Intentionalität der Mitteilung und damit die Kontrolle des Subjekts über seine eigenen Handlungen. ${ }^{8}{ }^{8}$ (168) So werden die von Birdy eingesetzten stimmlichen Gestaltungsmittel von Müller als Homologie zur »Realität« weiblicher Existenz »in einer durch Sexismus gekennzeichneten Welt« gedeutet, in der »Ertragen und Überwindung von vielfältigen Verletzungen « zum »Alltag vieler Frauen« (171) gehören.

Die unterschiedlichen Arten des Umgangs mit dem Klang der weiblichen Stimme, die in den vier von Sängerinnen performten Songs ausmacht wurden, verortet Müller abschließend als "Orientierungspunkte in einem musikästhetischen Feld außerhalb der sechten stimme« (173). Anders als in den vokalen Ausprägungen smännlicher Performanz ziele der Stimmklang in diesen sweiblichen Songs nicht auf »emotionale Kongruenz zwischen singendem Ich und Publikum « (Identifikation) ab; vielmehr stünden die Sängerinnen »in einer antagonistischen Position zum Publikum« (173), dem sie als »undurchschaubare Femme Fatale, als verfügbares Sexobjekt, als übernatürliche Mutter und als hilfloses Opfer« (174) gegenüberträten. Insofern hiermit in jedem Fall die Vorstellung einer objektifizierten Körperlichkeit verbunden sei, trage der Gebrauch der weiblichen Stimme im Popsong charakteristische Züge der Performanz von Weiblichkeit, wie sie auch in anderen gesellschaftlichen Sphären stattfinde (vgl. 175-177).

Hervorhebung original. 
'Sonischer und svokalischer Körper erscheinen demnach im Popsong geschlechtlich kodiert; sweiblicher Performanz im Popsong vollzieht sich nach diesem Verständnis als "Stimme des Anderen" (173), als klingender Ausdruck des Othering.

Müllers Buch widmet sich einem hochaktuellen, ja brisanten Thema mit Verve und Genauigkeit. Der sprachliche Stil ist ausgesprochen lesbar - alles andere als selbstverständlich angesichts der komplexen theoretischen Einbettung, die ja zahlreiche Forschungskontexte mit ihrem jeweils eigenen Begriffsrepertoire zusammenführt. Ausstattung und Aufmachung des Buchs sind ansprechend; allerdings hätte man dem sorgfältig aufbereiteten Inhalt ein genaueres Lektorat gewünscht. ${ }^{9}$ Aus musiktheoretischer Sicht wirft Müllers Buch eine Reihe von Fragen auf, von denen viele, doch nicht alle nach der Lektüre beantwortet sind. So ist hinsichtlich einer auf Überprüfbarkeit angelegten Analysemethodik zu fragen, ob die Arbeit den Herausforderungen, wie sie vom subjektiven Urteil abhängige Kategorien wie Assoziation und Homologie mit sich bringen, restlos zufriedenstellend begegnet. Ähnliches gilt für die systematische Einbeziehung des hörenden Subjekts in die zu untersuchenden Gegenstände, die Müllers Studie durch ein introspektives Vorgehen leistet, indem sie von der "eigenen Musikerfahrung " ausgeht und »versucht meine Eindrücke möglichst genau am Klang zu begründen « (115). Die autologische Komponente dieses Vorgehens wird explizit berücksichtigt (vgl. etwa 63 f.), doch entziehen sich im Unterbewussten angesiedelte Vorgänge, wie sie mit den Kategorien Genotext und rauditive Lüster angesprochen sind, per definitionem dem verifizierenden oder falsifizierenden Zugriff. Ob man den Verweis auf psychoanalytische Theoriebildung hier als ausreichende Absicherung der Untersuchungsergebnisse akzeptiert, dürfte seinerseits von sub-

9 Mehrere Zitate aus dem Originaltext erscheinen in dieser Rezension druckfehlerbereinigt (»Eindruck von Natürlichkeit«, 113; »eine starke Verbindung mit Weiblichkeit«, 158; »Femme Fatale«, 174). jektiven Überzeugungen abhängen. (Wer etwa wissenschaftstheoretisch voraussetzt, das menschliche Bewusstsein sei prinzipiell »intransparent « ${ }^{10}$ wird sich kaum zufriedengeben.) Allerdings reflektiert Müller selbst die Entscheidung für die Psychoanalyse als wissenschaftstheoretische Basis kritisch (vgl. 11, 76); zudem kann die Studie an bedeutende Vorgängerarbeiten im Bereich der Filmanalyse anschließen (vgl. 34). Tatsache bleibt, dass wiederholt auf Formulierungen wie »meines Erachtens« (150), »Meine persönliche Interpretation des Songs ist $[\ldots]$ (138) zurückgegriffen wird, die die Gültigkeit von Aussagen einschränken.

Positiv hervorzuheben ist in jedem Fall die Sorgfalt, mit der Müller der allenthalben lauernden Gefahr eines illegitimen Zugriffs auf fremde Bewusstseinsinhalte begegnet. Die hinter dem Stimmklang vermuteten sSubjektes werden konsequent als Projektionen behandelt (vgl. 148), der intentional fallacy somit aus dem Weg gegangen. Aussagen über die » wirkliche Intention der Sängerin" (138), „Kate Bushs sechte` Gefühle« (139), Birdys »bewusst[en] « (165) Einsatz stimmlicher Gestaltungsmittel vermeidet Müller ziemlich konsequent oder markiert sie zumindest als hypothetisch. ${ }^{11}$ Auch der Konstruktcharakter von Kategorien wie svokalischem nicht aus dem Blick; dafür sorgt schon die durchgängig präsente Reflexion der Produktionsbedingungen von Popmusik, unter denen das live erzeugte und technisch konservierte Klangereignis prinzipiell unbegrenzter Nachbearbeitung unterzogen werden kann (vgl. 94).

Zu diskutieren ist, ob die Annahme, in Popsongs werde Sexismus praktiziert und reproduziert, durch Müllers Studie als erwiesen betrachtet werden darf. In jedem Fall finden sich wenig Hinweise auf Versuche, Auswirkungen möglicher konzeptioneller Voreingenommen-

Luhmann 1995, 122.

11 Die Formulierung »Der bewusst intentionale Einsatz der Stimme« mit Bezug auf Bushs Aufnahme des Songs Feel It dürfte ein Lapsus linguae sein; unmittelbar zuvor erklärt Müller noch, die »angebliche /Wirklichkeit` von Kate Bushs Subjektivität» sei »nicht zugänglich» (140). 
heit, also Bestätigungsfehler, zu vermeiden. ${ }^{12}$ Von Interesse wäre etwa, 'weibliche` Popsongs, die Merkmale der sechten stimme aufweisen, aber eine Interpretation im Sinn von Kontrollverlust und/oder Objektifizierung nicht nahelegen, auf alternative Erklärungsmuster hin zu untersuchen. Hier müsste eine Interpretation als sselbstbewusste emotionale Mitteilung, (analog zu den smännlichen` Gegenparts) zumindest getestet und gegebenenfalls widerlegt werden. ${ }^{13}$ Jedenfalls erbringt die stichprobenartige Auswertung von zwei plus vier Songs nicht den Beweis dafür, dass in Popsongs durch den unterschiedlichen Einsatz smännlicher und 'weiblicher Stimmen generell akustisches Othering stattfinde. Eher kann die Studie als Aufforderung verstanden werden, Müllers (plausible) Angabe, die vorgenommene Songauswahl sei repräsentativ (vgl. 131), durch eigene Analysen weiterer Songs zu überprüfen. In diesem Zusammenhang ist mit Interesse auf die Ergebnisse von Müllers derzeitiger Forschung zu warten. ${ }^{14}$

Wichtige Anregungen hält Sound und Sexismus für künftige Entwicklungen des Fachs Musiktheorie bereit. Die Ansprüche, die von der rasanten Entwicklung der Performance Studies in jüngerer Zeit an die etablierten musikanalytischen Fachbereiche adressiert werden, stehen im Raum und sind nicht abzuweisen; die Auseinandersetzung mit dieser Herausforderung erscheint als Zukunftsaufgabe der Musiktheorie. ${ }^{15}$ Fragt man nach Möglichkeiten

12 Zum Problem des Bestätigungsfehlers vgl. Neuwirth/Rohrmeier 2016, 176 f.

13 Vgl. etwa die Ausführungen zu Taylor Swifts Song Shake it Off in Müller 2016, 9 f.

14 Vgl. https://jmueller.jimdofree.com/\%C3\%BCber -mich-about-me (5.4.2020).

15 Der Einzug von Methoden und Erträgen aus dem Bereich der Performance Studies in musiktheoretische Curricula ist aus dieser Sicht lebhaft zu begrüßen und als zukunftsweisend einzustufen. Vgl. etwa die Studienordnung für den MAStudiengang Musiktheorie an der Musikhochschule München, die eine eigene Lehrveranstaltung mit dem Titel Performance Studies vorsieht. Am Institut Interpretation der Hochschule der Künste Bern zählt zum Unterricht, der von der ersten im deutschsprachigen Raum eingerichteten Professur für Angewandte Interpretationsfor- der Vermittlung von Müllers Ansatz mit fachlichen Traditionen der Musiktheorie, so ist die Ausgangsentscheidung positiv zu vermerken, der zufolge sich das Augenmerk der Studie konsequent auf musikalische struktur (statt etwa ausschließlich auf Elemente des Rezeptionskontextes) richtet. So bietet Müllers Buch einer differenzierten Performanceanalyse von Vokalmusik nicht nur zahlreiche Anregungen, sondern auch ein beachtliches Repertoire von Kategorien zur Analyse des (Stimm-)Klangs als musikalischer Struktur. Die verschiedenen in Müllers Songanalysen beschriebenen Formen farblicher Modifikation des menschlichen Stimmklangs ('Knarrstimmes, 'Rufstimmes, Behauchung usw.) bilden den Grundstock für ein ausbaufähiges Instrumentarium, das im Analyse- wie im Gehörbildungsunterricht gewinnbringend auf weitere Popsongs, ebenso wie andere Formen vokalen Musizierens angewendet und dabei erweitert werden kann. Dass Müller Beobachtungen zum Stimmklang immer wieder mit analytischen Beobachtungen straditionelleren ¿ Zuschnitts verbindet und auf diese Weise zeigt, dass sie in den Kontext übergeordneter musikalischer Zusammenhänge eingeordnet werden können (vgl. besonders 160-164), ist im Hinblick auf die Vermittlung mit straditionellen strategien der Musikanalyse ebenfalls zu begrüßen.

Doch bietet Müllers Studie auch wichtige Hinweise für eine Musiktheorie, die sich systematisch für gesellschaftliche Kontexte öffnen möchte, etwa für das denkbare Projekt einer skulturwissenschaftlich informierten theorie bzw. Gehörbildung. ${ }^{16}$ Die Schwierigkeiten, die sich dem Vorhaben entgegenstellen, musikalische Struktur systematisch und robjektiven Kriterien folgend auf soziale Strukturen zu beziehen, werden an ihr in exemplarischer Weise deutlich, ebenso jedoch die Chancen eines solchen Unternehmens. Sound und Sexismus ermutigt also dazu, dieses Projekt weiter zu verfolgen.

\section{Kilian Sprau}

schung (seit 2011) angeboten wird, eine Art performancestilistisch informierte Gehörbildung.

16 Vgl. mit Bezug auf spopuläre Musikı den von Ismaiel-Wendt $(2011,15)$ formulierten Begriff einer »Postkoloniale[n] Gehörbildung«. 


\section{Literatur}

Berne, Peter (2008), Belcanto. Historische Aufführungspraxis in der italienischen Oper von Rossini bis Verdi. Ein praktisches Lehrbuch für Sänger, Dirigenten und Korrepetitoren, Worms: Wernersche Verlagssgesellschaft.

Cook, Nicholas (2013), Beyond the Score: Music as Performance, New York: Oxford University Press.

Ismaiel-Wendt, Johannes (2011), tracks'n'treks. Populäre Musik und Postkoloniale Analyse, Münster: Unrast.

Leech-Wilkinson, Daniel (2009), The Changing Sound of Music: Approaches to Studying Recorded Musical Performance, London: CHARM. http://www.charm.kcl.ac.uk/studies/ chapters/intro.html (5.4.2020)

Luhmann, Niklas (1995), »Was ist Kommunikation?», in: ders., Soziologische Aufklärung 6. Die Soziologie und der Mensch, Opladen: Westdeutscher Verlag, 113-124.

Müller, L. J. (2016), »Eih', Eih', Eih' Shake It $\mathrm{Oh}^{\prime}$. Popmusik als Konstruktionsort geschlechtsspezifischer Stimmen und Subjekte«, Betrifft Mädchen 1, 4-11.

Müller, L. J. (2017), »Hearing Sexism - Analyzing Discrimination in Sound", in: Popular Music Studies Today, hg. von Julia Merrill, Wiesbaden: Springer, 225-234. https://doi.org/10. 1007/978-3-658-17740-9_23 (5.4.2020)

Müller, L. J. (2018), »)l am only human? Ein Versuch weißes Begehren in populärer Musik zu analysieren«, in: Darüber hinaus... Populäre Musik und Überschreitung(en). 2. IASPM D-A-CH Konferenz Graz 2016, hg. von Stefa- nie Alisch, Susanne Binas-Preisendörfer und Werner Jauk, Oldenburg: BIS, 216-231.

Neuwirth, Markus / Martin Rohrmeier (2016), "Wie wissenschaftlich muss Musiktheorie sein? Chancen und Herausforderungen musikalischer Korpusforschung", Zeitschrift der Gesellschaft für Musiktheorie 13/2, 171-193. https://doi.org/10.31751/915 (5.4.2020)

Sadolin, Cathrine (2013), Complete Vocal Technique. Deutsche Ausgabe, übers. von Martin Carbow, Christa Wolf und Sebastian Kraft, Kopenhagen: Shout Publications.

Smith, Jacob (2008), Vocal Tracks. Performance and Sound Media, Berkeley (CA): University of California Press.

Soto-Morettini, Donna (2014), Popular Singing and Style, 2. Auflage, London: Bloomsbury.

Sprau, Kilian (2017), »Breit über mein Haupt dein schwarzes Haar. Vier auktoriale Versionen von Richard Strauss' SchackVertonung op. 19/2, betrachtet unter performativem Aspekt", Zeitschrift der Gesellschaft für Musiktheorie 14/2, 285-314. https://doi.org/10.31751/943 (5.4.2020)

Sprau, Kilian (i. V.), " '... an inborn architectural sense.... Theoretisch-methodologische Überlegungen zur Performance-Analyse am Beispiel einer Aufnahme von Maria Callas", in: Populäre Musik und ihre Theorien: Begegnungen - Perspektivwechsel - Transfers. 17. Jahreskongress der Gesellschaft für $\mathrm{Mu}$ siktheorie 2017, hg. von Christian Utz.

Walshe, Jennifer (2010), „Die Körnung der Stimme. Genealogien populärer Klangfarben«, MusikTexte 125, 57-59.

Sprau, Kilian (2020): L. J. Müller, Sound und Sexismus. Geschlecht im Klang populärer Musik. Eine feministisch-musiktheoretische Annäherung, Hamburg: Marta Press 2018. ZGMTH 17/1, 185-192.

https://doi.org/10.31751/1032

(C) 2020 Kilian Sprau (kontakt@kiliansprau.de)

Universität der Künste Berlin [Berlin University of Arts]

Dieser Text erscheint im Open Access und ist lizenziert unter einer Creative Commons Namensnennung 4.0 International Lizenz.

This is an open access article licensed under a

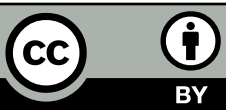

Creative Commons Attribution 4.0 International License.

eingereicht / submitted: 14/03/2020

angenommen / accepted: 14/03/2020 veröffentlicht / first published: 15/06/2020

zuletzt geändert / last updated: 15/06/2020 